\title{
La innovación psicosocial: planificar su implementación y difusión para prevenir la delincuencia juvenil
}

\section{The Psychosocial Innovation: Planification of Implementation and Dissemination to Prevent Juvenile Delinquency}

Recibido: diciembre 2 de 2011 | Revisado: marzo 8 de 2012 | Aceptado: abril 8 de 2012

\author{
LiNE LEBLANC* \\ MARIE ROBERT ** \\ Universidad de Quebec en Outaouais, Canadá
}

\begin{abstract}
RES U MEN
La innovación en la prevención de la delincuencia juvenil orienta sus acciones hacia el mejoramiento de la calidad de los ambientes sociales. Eso significa que las personas que interactúan en la vida cotidiana con los niños y adolescentes en dificultad en sus medios naturales (familia, escuela, barrio) se transforman en los grupos objetivo para participar en programas preventivos. Este enfoque recibe un sólido fundamento científico, pero su aplicación concreta está a menudo limitada debido a problemas en la difusión de la innovación psicosocial. Podemos fácilmente imaginar los obstáculos que puede haber para establecer un sistema eficaz de comunicación entre los diferentes grupos involucrados (investigadores, tomadores de decisión, agentes de intervención y miembros de la comunidad). Es importante reflexionar sobre este problema ya que la dificultad de difundir las nuevas prácticas en terreno puede poner en riesgo los efectos benéficos esperados. El presente texto tiene por objetivo el proponer un marco integrador orientado a optimizar el paso entre los universos científico, político y práctico apoyándose en la teoría de la difusión de la innovación.

Palabras clave autores

Teoría de la difusión de la innovación, prevención, delincuencia juvenil.

Palabras clave descriptores

Promoción y prevención, justicia juvenil, psicología social.

\section{A B S T R A C T}

Evidence-based psychosocial treatments for the prevention of juvenile delinquency emphasize the importance of a high quality social environment. However the promotion of this approach in real life is somewhat hindered by diffusion-related problems associated with psychosocial innovation. Indeed, communication between researchers, policy makers, clinicians and members of the community is not an easy task. The communication gap between research and practice is an important issue because its failure could compromise the impact of the intervention on youths at risk of juvenile delinquency. The objective of this article is to put forth a conceptual framework based on theory of diffusion of innovation specifically geared towards optimizing the cohesion between the scientific, politic and practice worlds. Key words authors

Prevention, Juvenile Delinquency, Theory of Diffusion of Innovation.

Key word plus

Promotion and Prevention, Juvenile Justice, Social Psychology.
\end{abstract}


En el campo de la intervención psicosocial se han desplegado muchos esfuerzos de investigación para identificar los programas eficaces. Este esfuerzo parece comprometido por un desequilibrio entre los avances en el conocimiento sobre la eficacia de los programas de prevención y de intervención y las adquiridas acerca de los mecanismos que permiten una implementación eficaz de aquellas (Stelk, 2006). Las investigaciones sobre la eficacia de los programas de intervención psicosocial son cruciales para mejorar los servicios ofrecidos, toda vez que estos conocimientos tienen un aporte limitado si no pueden superar las fronteras científicas y ser adoptados por los medios de práctica.

Así, paralelamente a un enfoque evaluativo basado en la noción de eficacia, se ve emerger, desde hace unos años, estudios evaluativos centrados en la noción de implementación. Investigaciones recientes muestran que la eficacia de los programas depende ampliamente de la manera en que se implementan (Aarons, Hurlburt \& Horwitz, 2011; Cousins, Aubry, Fowler \& Smith, 2004; Salyers et al., 2003). En otros términos, la eficacia de un programa o de una práctica no descansa únicamente en las cualidades intrínsecas, sino que es dependiente de la fase de implementación (Steckler, Goodman, McLeroy \& Koch, 1992).

La implementación está en el centro de un nuevo cuestionamiento, y permite identificar y comprender mejor las condiciones asociadas al éxito de esta fase determinante en el logro de los efectos benéficos esperados. La literatura científica reciente muestra que la implementación de programas o de prácticas ha sido abordada desde dos ángulos diferentes: el de la fidelidad y el de la innovación.

La evaluación de la fidelidad es particularmente pertinente bajo la óptica de difundir las mejores prácticas en medios diferentes y de asegurar que son aplicadas de manera homogénea y adecuada. La evaluación de la fidelidad o de la conformidad de un programa o de una práctica descansa esencialmente en un ejercicio de comparación entre lo que ha sido implementado y lo que debiera haber sido implementado. Los tests de fidelidad son concebidos para medir las diferencias entre los dos, de tal manera que el resultado final se interpreta en términos de grado o de nivel de implementación. Este indicador del éxito de la implementación limita la comprensión de los mecanismos que favorecen la adopción de la práctica propuesta por los agentes de intervención, que pueden influir en la fidelidad.

El enfoque centrado en la difusión de la innovación parece promisorio ya que puede esclarecer los resultados reducidos o decepcionantes constatados luego del análisis de la eficacia (Klein \& Knight, 2005). Estos estudios evaluativos buscan documentar el conjunto de las etapas inherentes a la implementación de una nueva práctica, a partir de su adopción hasta su fase de puesta en marcha. El grado de adhesión es el resultado investigado a través de comparaciones del grado de implementación en los estudios sobre la fidelidad. Este enfoque, asociado más que nada a la finalidad, significa que el interés debería más bien centrarse en los actores a los que se solicita implementar una nueva práctica, para examinar en qué medida esta es evaluada como potencialmente aceptable. Así, la pregunta es saber cómo los investigadores, los tomadores de decisiones, los agentes de intervención y las personas que interactúan con los jóvenes en dificultad en el medio natural, pueden articular sus acciones de manera coherente (Sterling \& Weisner, 2006).

El objetivo de este texto es proponer un marco integrador apoyado en la teoría de la difusión de la innovación (Rogers, 1995) que permita planificar mejor la fase de implementación de los programas considerados promisorios. En el presente trabajo se aplica este marco integrador en el ámbito de la prevención de la delincuencia juvenil, precisando los objetivos, las metodologías y las herramientas para cada grupo involucrado, sean los investigadores, los tomadores de decisiones, los agentes de intervención y el entorno de los jóvenes relacionado con problemas de comportamiento.

\section{Investigadores: determinar las mejores prácticas}

Los investigadores que se interesan en la prevención de la delincuencia juvenil proponen un enfoque que podemos calificar de innovador, ya que consiste en superponelas a las políticas orientadas al mejora- 
TABLA 1

La prevención de la delincuencia juvenil basada en datos probatorios

\begin{tabular}{|c|c|}
\hline Autores y títulos & Elementos del contenido \\
\hline $\begin{array}{l}\text { Eyberg, Nelson \& Boggs (2008). } \\
\text { Evidence-based psychosocial } \\
\text { treatments for children and ado- } \\
\text { lescents with disruptive behavior. }\end{array}$ & $\begin{array}{l}\text { La investigación sobre la intervención psicosocial basada en datos probatorios } \\
\text { pone en evidencia la importancia de la promoción y de la difusión de los procedi- } \\
\text { mientos psicológicos entre quienes interactúan directamente con los jóvenes en } \\
\text { dificultad. }\end{array}$ \\
\hline $\begin{array}{l}\text { Sukhodolsky \& Ruchkin (2006). } \\
\text { Evidence-Based Psychosocial } \\
\text { Treatments in the Juvenile Justi- } \\
\text { ce System. }\end{array}$ & $\begin{array}{l}\text { La intervención psicosocial entre jóvenes judicializados implica al joven mismo, } \\
\text { la familia y la comunidad. La aplicación de la intervención psicosocial a través del } \\
\text { sistema de justicia necesita una reflexión sobre la difusión y la implementación de } \\
\text { esta nueva práctica. }\end{array}$ \\
\hline $\begin{array}{l}\text { Welsh \& Farrington (2007). } \\
\text { Scientific support for early pre- } \\
\text { vention of delinquency and later } \\
\text { offending. }\end{array}$ & $\begin{array}{l}\text { Las intervenciones preventivas a múltiples niveles reciben un apoyo científico. Las } \\
\text { medidas preventivas debieran ser sobre: } \\
\text { - El enriquecimiento intelectual y el desarrollo de habilidades preescolares } \\
\quad \text { (individual) } \\
\text { - Las habilidades parentales en el contexto familiar } \\
\text { - El mejoramiento del medio escolar } \\
\text { - El mejoramiento del barrio } \\
\text { La transferencia de conocimientos debe hacerse localmente y ser apoyada por } \\
\text { políticas nacionales de prevención. }\end{array}$ \\
\hline
\end{tabular}

Fuente: elaboración propia.

miento de los ambientes sociales en los cuales los niños y adolescentes se desarrollan, tales como la familia, la escuela y el barrio (McCord, Widom \& Crowell, 2001). Apoyándose en datos probatorios, los investigadores han identificado las prácticas que se deben fomentar para prevenir la delincuencia juvenil y los medios en los cuales difundirlas. La Tabla 1 presenta las revisiones de literatura sobre esta temática.

Este enfoque preventivo se apoya en resultados provenientes de estudios longitudinales que han demostrado que el difícil perfil conductual de los niños afecta negativamente, desde las edades más tempranas, las relaciones que mantienen con los demás en los diferentes ámbitos de vida. Más recientemente, la interacción entre los factores individuales y ambientales, tales como el déficit neuropsicológico (Moffitt, 1993), el perfil conductual disruptivo (Broidy et al., 2003; Tremblay, Phil, Vitaro \& Dobkin, 1994), las prácticas educativas de los padres (Hoeve et al., 2008; Sampson \& Laub, 1990), el rechazo de los pares (Coie, 2004; Coie, Lochman, Terry \& Hyman, 1992), la asociación con pares desviados (Lacourse, Nagin, Tremblay, Vitaro \& Claes, 2003) y el clima social negativo de la escuela (LeBlanc, Swicher, Vitaro \& Tremblay,
2008), contribuyen al desarrollo y permanencia de los comportamientos antisociales durante la infancia y la adolescencia. Dicho de otra manera, es el ambiente social como conjunto de condiciones interpersonales el que viene a influir positiva o negativamente en las predisposiciones individuales y, de manera recíproca, entre los jóvenes con dificultades y las personas que interactúan en su vida cotidiana.

El estado actual de los conocimientos muestra que para evitar una trayectoria que lleva a la delincuencia juvenil incluso hasta la criminalidad adulta, hay que prevenir el cúmulo de experiencias relacionales negativas que pueden surgir en diferentes ambientes sociales. En efecto, el desarrollo de los comportamientos antisociales se produce a través de las reacciones provenientes de los padres, los hermanos, los pares, les profesores y el entorno. Por tanto, es importante sensibilizarlos sobre la importancia de adoptar la mejor práctica educativa posible, frente a los problemas de comportamiento tales como el oposicionismo, la hiperactividad y la agresividad. Esta perspectiva preventiva orienta las intervenciones hacia la sensibilización y la formación de las personas que interactúan en la vida cotidiana con los niños y los adolescentes con dificultades, lo que implica un enfoque en múlti- 
ples niveles (Eyberg et al., 2008; Van Der Merwe $\&$ Dawes, 2007).

La implementación de programas centrados en la calidad del ambiente es de hecho compleja, ya que implica a todo el sistema social, y demasiado a menudo se observa que su difusión no es la óptima (Hawkins, Shapiro \& Fagan, 2010). Una de las razones que explica esta dificultad es la ausencia de infraestructura de prevención en la comunidad. Para disminuir la brecha entre el estado de los conocimientos y el de las prácticas, la primera etapa consiste no solo en proponer políticas y planes de acción en concordancia con los datos probatorios, sino en establecer las condiciones que puedan asegurar una difusión eficaz de la innovación.

\section{Tomadores de decisión: difundir la innovación psicosocial}

Según Rogers (1995), la innovación es una idea, una práctica o un objeto percibido como nuevo por los individuos. Por su lado, la difusión es un proceso por el cual la innovación es transmitida o propagada a través de ciertos canales de comunicación entre los miembros de un sistema social. Los tomadores de decisión tienen la responsabilidad de planificar la crucial fase de la difusión de la innovación psicosocial con quienes serán los que la pongan en práctica. No es suficiente con producir una política o un plan de acción gubernamental para que la integración de las nuevas prácticas se actualice en los medios de intervención. La adhesión del personal ante la nueva práctica que deberá ser implementada necesita de un tiempo más o menos largo, y está fuertemente determinada por las estrategias de difusión (informar y persuadir) puestas en marcha respecto de la nueva práctica.

Desde el punto de vista de la teoría de Rogers (1995), para comprender bien la evolución de la opinión sobre la innovación, hay que considerar la diferencia entre su adopción y su implementación (Klein \& Knight, 2005). El primer concepto se relaciona con la toma de decisión respecto de una nueva práctica (concientización, persuasión y decisión), en tanto que la segunda implica necesariamente su utilización en el terreno (implemen- tación y confirmación). Dicho con más precisión, la implementación de una nueva práctica lleva a tres fases específicas: la formación del personal, la adopción de la innovación y su puesta en marcha (Rogers, 1995). A cada una de estas tres fases están asociados obstáculos que pueden perjudicar la evolución del proceso de implementación.

Una planificación deficiente de la difusión de una nueva práctica puede dar lugar a concepciones erróneas o a un conocimiento superficial, incompleto e insuficiente de la misma y compromete así su implementación. Su desconocimiento hará difícil la etapa subsecuente de su adopción. Así, los tomadores de decisión deben preocuparse de la comprensión de la práctica innovadora por parte de quienes tendrán que implementarla, de las percepciones positivas en cuanto a su adecuación y eficacia, así como de la motivación para implementarla (Pagoto et al., 2007). A pesar de lograr una buena comprensión de una nueva práctica, es posible que la percepción de falta de recursos o de capacidades del medio o del personal para ponerla en marcha, pueda frenar su adopción.

Para facilitar la planificación de la difusión de la innovación por quienes tienen la responsabilidad de hacerlo, Hall y Hord (2001) proponen el Concerns Based Adoption Model que permite planificar las acciones en tres tiempos. La etapa preadopción hace énfasis en lo previo y en cómo la innovación va a afectar el trabajo de quienes la adopten. La etapa inicial se relaciona con la adecuación entre la innovación y el trabajo en la vida cotidiana, y la etapa de consolidación se centra en la retroalimentación que se debe dar en relación con las consecuencias de la innovación en la autonomía profesional.

Una revisión exhaustiva de la literatura realizada por Greenhalgh, Robert, Macfarlane, Bate y Kyriakidou (2004) enriquece el concepto de difusión de la innovación, agregándole el concepto de difusión, que lleva a una planificación más activa en cuanto a los esfuerzos de persuasión para promoverla y el concepto de durabilidad, que se refiere a la integración de la innovación en la rutina. Los trabajos de Greenhalgh et al. han permitido poner en evidencia que la perspectiva contextual de la difusión de la innovación puede frenarla o facilitar- 
la. En otras palabras, los primeros estudios privilegiaron un nivel individual de análisis; los estudios recientes tienden al examen de la concordancia entre la innovación y el sistema en el cual esta se implementa. Este enfoque muestra la importancia de compartir la responsabilidad de la adhesión a una nueva práctica entre quienes toman decisiones y los equipos de intervención.

\section{Equipos de intervención: adoptar las prácticas innovadoras}

Los datos probatorios son cada vez más utilizados para establecer políticas a gran escala, y en este contexto quienes intervienen son los llamados a implementar nuevas prácticas. Así, se estudia una tasa elevada de adhesión a la práctica innovadora, que puede variar principalmente en función de las percepciones sobre las características innovadoras, del impacto sobre la clientela y del contexto de trabajo.

La adopción de una nueva práctica implica mecanismos de difusión por medio de los cuales quienes intervienen la conocen y adquieren una comprensión adecuada, que respalde su opinión favorable o desfavorable. Cinco atributos propios de la innovación parecen modular la fase de adopción de una nueva práctica. En otras palabras, la adopción por quienes intervienen, a quienes se solicita implementar una nueva práctica, será más fácil y más rápida si esta posee las siguientes características (Rogers, 1995):

a) Ventaja relativa: el grado según el cual la innovación (o la práctica nueva) es percibida como superior a la práctica reemplazada.

b) Compatibilidad: el grado según el cual la nueva práctica es percibida como coherente con los valores, las experiencias y las necesidades de los individuos susceptibles de adoptarla.

c) Complejidad: el grado según el cual la nueva práctica es percibida como difícil de comprender o de utilizar. Algunos autores (Stelk, 2006) agregan también su flexibilidad: 'la medida en que se adapta a diversas situaciones'. d) Reversibilidad (puesta a prueba): la posibilidad de que la innovación pueda ser experimentada y adaptada.

e) Observabilidad: el grado según el cual los resultados de la innovación se hacen visibles.

La percepción positiva de estos atributos se corresponde de alguna manera con los esfuerzos por consentir en implementar una nueva práctica, con el objetivo de generar cambios deseables para las personas que serán expuestas a ella.

La teoría de la difusión de la innovación motiva a quienes toman decisiones a considerar las necesidades de las personas que la implementarán (Pagoto et al., 2007), y a establecer estrategias orientadas a informar, sostener y dar la posibilidad a quienes intervienen de evaluar la pertinencia de la práctica que se desea promover. Hay que recordar aquí que la novedad de una práctica no significa necesariamente que ella será pertinente en el contexto real. En efecto, la evaluación de la implementación puede hacer surgir importantes lagunas que pueden llevar a un cuestionamiento de la práctica propuesta. Así, la evaluación de la implementación puede también servir para poner en evidencia las malas prácticas. Por ejemplo, Leone (2008) examinó la aplicación a nivel regional de una política nacional sobre el consumo de drogas con fines personales. Se trata de una política basada en mecanismos de disuasión mediante la amenaza de represalias jurídicas. La evaluación de la implementación de este programa reveló una distancia considerable entre las medidas fomentadas por quienes toman decisiones y las implementadas en el contexto real. Así, una nueva práctica que es mal percibida por quienes intervienen, y que parece inapropiada en su contexto de trabajo, tiene pocas posibilidades de ser utilizada luego de su implementación.

Los estudios evaluativos que utilizan el marco teórico de Rogers indican que la adopción de una nueva práctica no está solo motivada por la calidad de los atributos, sino también por los efectos terapéuticos anticipados. Por ejemplo, el estudio realizado por Aarons y Palinkas (2007) demuestra que la implementación eficaz de las prácticas basadas en datos probatorios, orientados a reducir la 
negligencia en relación con los niños, depende de seis condiciones: la aceptación de la práctica innovadora por quien interviene y por la familia, su pertinencia para ayudar a las familias, la motivación de quien interviene para integrarla en su práctica, la experiencia frente a esta nueva práctica, el apoyo organizacional ofrecido a quienes intervienen y el impacto de esta práctica sobre el servicio en cuanto a proceso y resultado. Un estudio cualitativo realizado por Nelson y Steele (2006), ha mostrado que las prácticas que se distinguen por su aplicabilidad, su consideración de la relación terapéutica y la posibilidad de modificar el protocolo de intervención, pueden estimular más a quienes intervienen en la adopción.

Además de evaluar los atributos de una nueva práctica y su impacto positivo para la clientela, hay que considerar las características del entorno de trabajo en el cual se implementa la práctica. A este respecto, los trabajos de Fuller et al. (2007) muestran la influencia de la apertura al cambio, en las organizaciones, sobre la opinión de quienes intervienen en la utilización de las mejores prácticas. La adhesión a ellas es mayor en los entornos donde el personal percibe una necesidad de mejoramiento de los programas aplicados, un mejor acceso a Internet, un nivel más alto de influencia de los pares, más oportunidades para el desarrollo profesional, un sentido agudo de la misión organizacional y más presión organizacional. En el mismo sentido, el estudio desarrollado por Simpson y Flynn (2007) indica que la percepción de quienes intervienen respecto de la necesidad de los programas, la apertura de la organización en relación con el cambio, la calidad de la formación continua y el compromiso de las personas objetivo de las intervenciones, son factores críticos. Otro estudio demuestra que la discontinuidad de las prácticas innovadoras en salud mental está relacionada con la ausencia de recursos financieros y también con los problemas para atraer y retener un personal calificado (Massatti, Sweeney, Panzano \& Roth, 2008).

La teoría de la difusión de la innovación propone que una percepción positiva de la práctica innovadora y de su impacto para la clientela, combinada con las condiciones contextuales propicias, induce a quienes intervienen a adherirse a ellas y a implementarlas con el rigor necesario. Por ejemplo, un estudio comparativo sobre la implementación en dos diferentes sitios de un sistema para mejorar la salud mental de los niños y adolescentes, puso en evidencia un mejoramiento para los niños y adolescentes que han recibido el servicio, y se observó más en un lugar que en el otro (Foster, Stephens, Krivelyova \& Gamfi, 2007). Este estudio muestra que las características relacionadas con la implementación pueden explicar las diferencias en los resultados obtenidos. Así, las mejores prácticas exigen las mejores implementaciones para llegar a los resultados esperados.

\section{El entorno de los jóvenes: interactuar contando con apoyo social}

La adhesión de quienes intervienen en el enfoque preventivo centrado en la calidad de los entornos sociales, debe necesariamente ser seguida de la participación activa de los padres, profesores, pares y miembros de la comunidad en los programas de prevención ofrecidos. En efecto, las personas que interactúan con los niños con dificultades en los entornos naturales son las llamadas a adoptar una nueva manera de ver y de apoyar a los jóvenes con problemas de comportamiento, lo cual podría romper el ciclo de interacciones negativas.

Para ayudar a las personas que interactúan en la vida cotidiana con los niños y adolescentes con problemas de comportamiento, la teoría de la autodeterminación desarrollada por Deci y Ryan (2002) es de utilidad. Este enfoque se orienta a actualizar el potencial de los jóvenes a través del apoyo social. Se trata de enseñar a las personas que interactúan con los jóvenes con dificultades a satisfacer tres necesidades psicológicas universales: la autonomía (toma de decisiones frente a los comportamientos), la competencia (capacidad de lograr un objetivo definido) y la pertenencia (sentirse conectado a los otros de manera afectuosa, cálida y positiva). Precisamente, el apoyo a la autonomía implica una actitud de respeto, favorecer la elección más que la presión y alentar el compromiso personal. El apoyo a la competencia se observa en una comunicación 
TABLA 2

Marco integrador para la difusión de la innovación psicosocial

\begin{tabular}{|c|c|c|c|c|}
\hline Actores & Investigadores & $\begin{array}{c}\text { Tomadores de } \\
\text { decisiones }\end{array}$ & Agentes de intervención & Entorno \\
\hline Objetivos & $\begin{array}{l}\text { Determinar las mejores } \\
\text { prácticas y evaluar } \\
\text { no solo la eficacia } \\
\text { sino también la } \\
\text { implementación }\end{array}$ & $\begin{array}{l}\text { Difundir la innovación } \\
\text { psicosocial e establecer } \\
\text { las condiciones para la } \\
\text { implementación }\end{array}$ & $\begin{array}{l}\text { Adoptar la innovación } \\
\text { psicosocial }\end{array}$ & $\begin{array}{l}\text { Interactuar } \\
\text { adecuadamente con los } \\
\text { jóvenes en dificultad }\end{array}$ \\
\hline Metodologías & $\begin{array}{l}\text { Evaluación de la } \\
\text { implementación y } \\
\text { metaanálisis de las } \\
\text { mejores prácticas }\end{array}$ & $\begin{array}{l}\text { Evaluación de los } \\
\text { mecanismos de difusión } \\
\text { de la innovación }\end{array}$ & $\begin{array}{l}\text { Evaluación de las } \\
\text { características de la } \\
\text { práctica innovadora }\end{array}$ & $\begin{array}{l}\text { Evaluación de la } \\
\text { participación de } \\
\text { las personas en } \\
\text { los programas de } \\
\text { prevención }\end{array}$ \\
\hline Herramientas & $\begin{array}{l}\text { Síntesis de las mejores } \\
\text { prácticas, programas } \\
\text { eficaces }\end{array}$ & $\begin{array}{l}\text { Estrategias de difusión } \\
\text { de la innovación } \\
\text { (Rogers, 1995) }\end{array}$ & $\begin{array}{l}\text { Guía de análisis de las } \\
\text { características de la } \\
\text { práctica innovadora } \\
\text { (Rogers, 1995) } \\
\text { y del contexto en el } \\
\text { cual es implementado }\end{array}$ & $\begin{array}{l}\text { Apoyo a la autonomía, } \\
\text { la competencia y la } \\
\text { pertenencia de parte de } \\
\text { los padres, profesores } \\
\text { y miembros de la } \\
\text { comunidad } \\
\text { Deci y Ryan (2002) }\end{array}$ \\
\hline \multicolumn{5}{|c|}{ Resultados esperados para los niños y adolescentes en riesgo: adaptación psicosocial } \\
\hline
\end{tabular}

Fuente : elaboración propia.

eficaz de la información, una retroalimentación personalizada, un nivel de desafío óptimo y un aprendizaje activo. El apoyo a la pertenencia se basa en el establecimiento de relaciones interpersonales positivas y la transmisión de valores (Green-Demers, 2006). Es esperable que un ambiente social que apoya estas tres necesidades incremente el desarrollo y el ajuste psicológico de las personas.

Un estudio cualitativo desarrollado por Sander, Sharkey, Olivarri, Tanigawa y Mauseth (2010), permite demostrar que los adolescentes con problemas de comportamiento tienen necesidad de establecer relaciones basadas en el apoyo (aceptación y no enjuiciamiento), pese a las consecuencias de sus conductas. Los autores mencionan la pertinencia de la teoría de la autodeterminación para aportar un apoyo social a estos jóvenes en dificultades. Así, las personas que interactúan con estos jóvenes podrían ser sensibilizadas al impacto posible del apoyo social sobre la adaptación psicosocial. Este enfoque es complementario con los programas específicos de prevención de la delincuencia juvenil, orientados al mejoramiento de los entornos sociales.
El mejoramiento de los entornos sociales involucra a todo el sistema, lo que exige una planificación rigurosa de todas las etapas de implementación de una política social. Puede ser útil apoyarse en un marco integrador que favorezca los canales de comunicación entre todos los grupos de actores, ya sea de los investigadores, de los que toman decisiones, de los agentes que intervienen y de las personas que interactúan con los jóvenes con problemas de comportamiento. La Tabla 2 presenta una síntesis de los objetivos, metodologías y herramientas que podrían estructurar de manera más coherente los esfuerzos acordados por estos grupos.

\section{Conclusión}

El presente texto propone el encuentro entre el saber científico y el saber experiencial, con el objetivo de mejorar la implementación y la difusión de los programas de prevención de la delincuencia juvenil. Aunque los objetivos y los medios hayan sido precisados con la ayuda de un marco integrador, el interrogante sobre la coordinación entre los 
grupos implicados deberá ser objeto de una atención permanente.

Hay una voluntad, en muchos países, de proponer políticas y planes de acción gubernamentales que se apoyen en datos probatorios. Este enfoque es sin duda un paso en la dirección correcta para disminuir la brecha entre el estado de los conocimientos y el estado de las prácticas. Aún más, hay que retener la idea de que la trayectoria de la difusión de la innovación psicosocial es un proceso complejo con escollos que pueden hacer difícil la cohesión entre investigadores, tomadores de decisiones, los agentes intervinientes y las personas provenientes del medio natural. Para poder comprender mejor lo que puede comprometer la difusión de la innovación psicosocial, las futuras investigaciones evaluativas deberían orientarse no solo hacia la eficacia, sino también hacia la implementación.

Las investigaciones sobre la implementación de nuevas prácticas deberían dar una atención particular al grado de adhesión por parte de quienes intervienen y a las condiciones que la facilitan. Los protocolos de investigación que tienen por objetivo evaluar el proceso que lleva a la adopción de una nueva práctica debería considerar la complejidad del proceso, examinando los factores que explican la variación de las tasas de adhesión a la innovación, la presencia de innovaciones múltiples sobre una política social, la diferencia entre quienes intervienen adoptando rápidamente la innovación y quienes la adoptan tardíamente (Meyer, 2004). Un elemento interesante que se debe profundizar es la relación entre la motivación de quienes intervienen y los efectos positivos anticipados por la clientela.

La evaluación de la implementación de los programas o prácticas puede transformarse en una oportunidad para facilitar una difusión de los conocimientos en los medios de práctica. Dado que son los investigadores quienes, en última instancia, analizan los datos de la implementación, es probable que los participantes no conozcan los resultados de la implementación. Justificar este hecho apoya el marco integrador propuesto aquí, que se refiere a la aplicación de un proceso de decisiones que va de la identificación de las mejores prácticas a su integración en la rutina del entorno.
Lo que hay que retener de este texto es que la coherencia entre los resultados de las investigaciones, las políticas gubernamentales y la implementación de los programas reconocidos como eficaces, es posible en la medida en que los canales de comunicación entre los grupos involucrados estén bien establecidos y que se nombre alguna instancia como responsable de la difusión y de la implementación de la innovación psicosocial. Así, todos los esfuerzos serán orquestados para optimizar los efectos benéficos entre las personas con dificultades o en riesgo.

\section{Referencias}

Aarons, G. A., Hurlburt, M. \& Horwitz, S. M. (2011). Advancing a conceptual model of evidence-based practice implementation in public service sectors. Administration and Policy in Mental Health and Mental Health Services Research, 38(1), 4-23.

Aarons, G. A. \& Palinkas, L. A. (2007). Implementation of evidence-based practice in child welfare: Service provider perspectives. Administration and Policy in Mental Health and Mental Health Services Research, 34(4), 411-419.

Broidy, L. M., Nagin, D. S., Tremblay, R. E., Bates, J. E., Brame, B., Dodge, K. A., et al. (2003). Developmental trajectories of childhood disruptive behaviors and adolescent delinquency: A six-site, cross-national study. Developmental Psychology, $39(2), 222-245$.

Coie, J. D. (2004). The impact of negative social experiences on the development of antisocial behavior. In J. B. Kupersmidt \& K. A. Dodge (Eds.), Decade of behavior. Children's peer relations: From development to intervention (pp. 243-267). Washington: American Psychological Association.

Coie, J. D., Lochman, J. E., Terry, R. \& Hyman, C. (1992). Predicting early adolescent disorder from childhood aggression and peer rejection. Journal of Consulting and Clinical Psychology, 60(5), 783-792.

Cousins, J. B., Aubry, T. D., Fowler, H. S. \& Smith, M. (2004). Using key component profiles for the evaluation of program implementation in intensive mental health case management. Evaluation and Program Planning, 27(1), 1-23. 
Deci, E. L. \& Ryan, R. M. (2002). Intrinsic motivation and self-determination in human behavior. New York: Plenum Press.

Eyberg, S. M., Nelson, M. M. \& Boggs, S. R. (2008). Evidence-based psychosocial treatments for children and adolescents with disruptive behavior. Journal of Clinical Child and Adolescent Psychology, 37(1), 215-237.

Foster, E. M., Stephens, R., Krivelyova, A. \& Gamfi, P. B. (2007). Can system integration improve mental health outcomes for children and youth? Children and Youth Services Review, 29(10), 1301-1319.

Fuller, B. E., Rieckmann, T., Nunes, E. V., Miller, M., Arfken, C., Edmundson, E., et al. (2007). Organizational Readiness for Change and opinions toward treatment innovations. Journal of Substance Abuse Treatment, 33(2), 183-192.

Green-Demers, I. (2006, enero). The snowball effect of social support: Cumulative influence of teachers, parents and friends on academic motivation. Trabajo presentado en I Annual Meeting of the Society for Personality and Social Psychology, Palm Springs, California.

Greenhalgh, T., Robert, G., Macfarlane, F., Bate, P. \& Kyriakidou, O. (2004). Diffusion of innovations in service organization: Systematic review and recommendations. The Milbank Quarterly, 82(4), 581-629.

Hall, G. E. \& Hord, S. M. (2001). Implementing change: Patterns, principles, and potholes. Boston: Allyn and Bacon.

Hawkins, J. D., Shapiro, V. B. \& Fagan, A. A. (2010). Disseminating effective community prevention practices: Opportunities for social work education. Research on Social Work Practice, 20(5), 518-527.

Hoeve, M., Blokland, A., Dubas, J. S., Loeber, R., Gerris, J. R. M. \& van der Laan, P. H. (2008). Trajectories of delinquency and parenting styles. Journal of $A b$ normal Child Psychology, 36(2), 223-235.

Klein, K. J. \& Knight, A. P. (2005). Innovation implantation: Overcoming the challenge. Current Directions in Psychological Science, 14(5), 243-246.

Lacourse, E., Nagin, D. S., Tremblay, R. E., Vitaro, F. $\&$ Claes, M. (2003). Developmental trajectories of boys' delinquent group membership and faci- litation of violent behaviors during adolescence. Development and Psychopathology, 15(1), 183-197.

LeBlanc, L., Swischer, R., Vitaro, F. \& Tremblay, R. E. (2008). High school social climate and antisocial behaviors: A 10 year longitudinal and multilevel study. Journal of Research on Adolescence, 18(3), 395-419.

Leone, L. (2008). Realistic evaluation of an illicit drug deterrence programme: Analysis of a case study. Evaluation, 14(1), 9-28.

Massatti, R. R., Sweeney, H. A., Panzano, P. C. \& Roth, D. (2008). The de-adoption of innovative mental health practices (IMHP): Why organizations choose not to sustain an IMHP. Administration and Policy in Mental Health and Mental Health Service Research, 35(1-2), 50-65.

McCord, J., Widom, C. \& Crowell, N. (2001). Juvenile crime, Juvenile Justice. Panel on juvenile crime: Prevention, treatment and control. Washington: National Academy Sciences.

Meyer, G. (2004). Diffusion methodology: Time to innovate? Journal of Health Communication, 9(1), 59-69.

Moffitt, T. E. (1993). Adolescence-limited and life-course-persistent antisocial behavior: A developmental taxonomy. Psychological Review, 100(4), 674-701.

Nelson, T. D. \& Steele, R. G. (2006). Beyond efficacy and effectiveness: A multifaceted approach to treatment evaluation, professional psychology. Research and Practice, 37(4), 389-397.

Pagoto, S. L., Spring, B., Coups, E. J., Mulvaney, S., Coutu, M. -F. \& Ozakinci, G. (2007). Barriers and facilitators of evidence-based practice perceived by behavioral science health professionals. Journal of Clinical Psychology, 63(7), 695-705.

Rogers, E. M. (1995). Diffusion of innovations (4th. ed.). New York: Free Press.

Salyers, M. P., Bond, G. R., Teague, G. B., Cox, J. F., Smith, M. E., Hicks, M. L., et al. (2003). Is It ACT Yet? Real-world examples of evaluating the degree of implementation for assertive community treatment. The Journal of Behavioral Health Services \& Research, 30(3), 304-320.

Sampson, R. J. \& Laub, J. H. (1990). Crime and deviance over the life course: The salience of adult social bonds. American Sociological Review, 55(5), 609-627. 
Sander, J. B., Sharkey, J. D., Olivarri, R., Tanigawa, D. A. \& Mauseth, T. (2010). A qualitative study of juvenile offenders, student engagement, and interpersonal relationships: Implications for research directions and preventionist approaches. Journal of Educational \& Psychological Consultation, 20(4), 288-315.

Simpson, D. D. \& Flynn, P. M. (2007). Moving innovations into treatment: A stage-based approach to program change. Journal of Substance Abuse Treatment, 33(2), 111-120.

Steckler, A., Goodman, R., McLeroy, K., Davis, S. \& Koch, G. (1992). Measuring the diffusion of innovative health promotion programs. American Journal of Health Promotion, 6(3), 214-224.

Stelk, W. J. (2006). Implementing health-care innovations: In search of a theoretical foundation for a science of implementation. International Journal of Mental Health, 35(2), 35-49.
Sterling, S. \& Weisner, C. (2006). Translating research findings into practice: Example of treatment services for adolescents in managed care. Alcohol Research \& Health, 29(1), 11-18.

Sukhodolsky, D. G. \& Ruchkin, V. (2006). Evidencebased psychosocial treatments in the juvenile justice system. Child and Adolescent Psychiatric Clinics of North America, 15(2), 501-516.

Tremblay, R. E., Pihl, R. O., Vitaro, F. \& Dobkin, P. L. (1994). Predicting early onset of male antisocial behavior from preschool behavior. Archives of General Psychiatry, 51(9), 732-739.

Van der Merwe, A. \& Dawes, A. (2007). Youth violence: A review of risk factors, causal pathways and effective intervention. Journal of Child and Adolescent Mental Health, 19(2), 95-113.

Welsh, B. C. \& Farrington, D. P. (2007). Scientific support for early prevention of delinquency and later offending. Victims \& Offenders, 2(2), 125-140. 